\title{
Efficacy of a Diarylheptanoid Derivative against Leishmania amazonensis
}

\author{
Luciana Vignólio Alves, Rosane Maria Temporal, Léa Cysne-Finkelstein, \\ Leonor Laura Leon ${ }^{+}$
}

Departamento de Imunologia, Instituto Oswaldo Cruz-Fiocruz, Av. Brasil 4365, 21045-900 Rio de Janeiro, RJ, Brasil

The activity of several diarylheptanoid derivatives (curcuminoids) was previously evaluated against Leishmania amazonensis promastigotes and among them the most active compound was the [1-(4-methoxy-phenyl)-7-(3,4methoxy-4-hydroxy-phenyl)-1,6-heptadien-3, 5-dione]. This derivative was chosen to be assayed in vivo in a treatment trial. For these experiments, the curcuminoid compound was used in a concentration equivalent to the $I C_{50}$ $24 \mathrm{~h}$, obtained from the previous study. Balb/c mice were inoculated subcutaneously in the footpad with L. amazonensis infective promastigotes and 4 weeks after the inoculation, the animals were treated with different schemes, varying from 1 to 3 doses. In all the experiments, Pentamidine Isethionate was used as reference drug under the same experimental conditions. The results showed that one dose was not enough to heal the lesion, however, with 2 and 3 doses the efficiency of the assayed compound was clear. On the other hand, treatment with Pentamidine Isethionate using the three different schemes was not satisfactory when compared to the curcuminoid derivative.

Key words: diarylheptanoid - treatment trial - Leishmania amazonensis - leishmaniasis

Leishmania species are pathogenic protozoa that infect man and cause considerable morbidity and mortality, currently are an increase in risk factors for leishmaniasis worldwide (Desjeux 2001). Current therapy for leishmaniasis is unsatisfactory; the actual treatments show a high toxicity and are not fully effective, thus the search to develop new chemotherapy for the treatment is urgent. Previous work of our laboratory described the in vitro and in vivo effectiveness of some diarylheptanoids extracted from Centrolobium sclerophyllum (Leguminosae) and semi-synthetic derivatives against Leishmania amazonensis (Araújo et al. 1998, 1999, Gomes et al. 2002). These compounds are analogous of curcumin (diferuloylmethane), a yellow odourless pigment isolated from the rhizome of turmeric (Curcuma longa L., Zingiberaceae) a natural product very studied which showed anti-inflammatory, anti-human immunodeficiency virus, anti-bacteria and anti-oxidant effects and recently were related as excellent anti-protozoal drug mainly against $L$. amazonensis (Araújo \& Leon 2001, Koide et al. 2002, Saleheen et al. 2002). Continuing our studies with these classes of compounds, new diarylheptanoid derivatives were completely synthesized and tested for activity against L. amazonensis. L. braziliensis and L. chagasi promastigotes and L. amazonensis axenic amastigotes. Now, the most effective compound from the in vitro assay is being used in a treatment trial against cutaneous leishmaniasis caused by L. amazonensis. Our results are promising and a challenge to continue the search for new anti-leishmanial drugs.

${ }^{+}$Corresponding author. Fax: $+55-21-2280.1496$. E-mail: 1leon@ioc.fiocruz.br

Received 9 October 2002

Accepted 25 March 2003

\section{MATERIALS AND METHODS}

Parasites - L. amazonensis infective promastigotes (MHOM/BR/77/ LTB0016 strain) were used. Parasites were grown at $26^{\circ} \mathrm{C}$ in Schneider's Drosophila medium supplemented with $10 \%(\mathrm{v} / \mathrm{v})$ heat-inactivated fetal calf serum (FCS). Cells were harvested in the late log phase, resuspended in fresh medium, counted in Neubauer's chamber and adjusted to a concentration of $1 \times 10^{7}$ parasites $/ 50 \mu 1$.

Animals - Age-matched 4- to 6-weeks-old male Balb/c mice, weighing 20-25 g was used in this study. Five mice were included in each group of treatment or control group and inoculated subcutaneously in the footpad with $1 \times 10^{7}$ parasites $/ 50 \mu \mathrm{l}$. After 4 weeks of inoculation, mice were treated in different schemes with a drug concentration equivalent to the $\mathrm{IC}_{50} / 24 \mathrm{~h}=514 \mathrm{ng} / \mathrm{ml}$ (for diarylheptanoid) and $4 \mathrm{mg} / \mathrm{kg}$ of body weight for Pentamidine, which is a concentration equivalent to those used in the clinic of leishmaniasis. For the experimental handling, the groups had been divided as follows: group 1, received 1 dose (D1 and P1 for diaryl derivative and Pentamidine Isehionate, respectively); group 2, received 2 doses (D2 and P2); group 3, received 3 doses (D3 and P3), both with a 15 days interval time of each dose, plus a group of infected mice (CI), which was left untreated and a group of non-infected mice $(\mathrm{CN})$. Lesion sizes were estimated weekly for 12 weeks post-infection.

Drug treatment - Compound [1-(4-methoxy-phenyl)7-(3,4-methoxy-4-hydroxy-phenyl)-1,6-heptadien-3, 5dione] is a curcuminoid analogue synthesized and kindly donated by AF Arrieta of the Technique Leipzig University, Germany. Treatments were initiated four weeks after inoculation when the infection was well established and lesions were obvious. Pentamidine Isethionate (May \& Baker Lab., England) was used as reference drug. Diarylheptanoid and Pentamidine Isethionate were dissolved in $50 \mu \mathrm{l}$ of Schneider's medium and administrated as described above. 
Statistical analysis - Significance was determined using a non-paired $t$ student test. Differences were considered to be significant when $\mathrm{P}<0.05$. Each experiment was done in triplicate.

\section{RESULTS AND DISCUSSION}

Several factors would be associated with the noneffectiveness of anti-leishmanial drugs. First of all, it has to be considered the fact that parasites are protected inside macrophages leading to a deficiency for the drug to reach them. Furthermore, in the way to reach the parasites the compounds would suffer some changes in its structure and for that reason, resulting in significant difference in the activity on promastigotes and amastigotes. One good example of it is related to the pentavalent antimonials, which are not effective against promastigotes and within the host cell are changed to trivalent and under this form are very active against amastigotes, despite its toxicity. Concerning to diarylhepatnoid derivatives we already described these difference in their activity on those leishmania evolutive forms. Furthermore, these data showed that, in general, diaryl derivatives are efficient against Leishmania species without toxicity to peritoneal macrophages and no inflammatory reaction in the area where the drug was injected (Araujo et al. 1999, Alves et al. 2003).

Although there are several compounds being studied, it is important to search for new compounds with high toxicity and low toxicity. In this work, a treatment trial was realized using a [1-(4-methoxy-phenyl)-7-(3,4methoxy-4-hydroxy-phenyl)-1,6-heptadien-3, 5-dione], a diaryl derivative which was the most effective in the in vitro assay. The compound was used in three doses injected in mice previously infected with $L$. amazonensis and having Pentamidine Isethionate as reference drug (Fig. 1 shows the chemical structure of the compounds). The follow up of the experiment (up to 8 weeks) showed a highly significant decrease $(\mathrm{p}<0.001)$ in the lesion size when compared to the control group (CI) or even with those groups of mice treated with Pentamidine Isethionate
(P1, P2 and P3). Also, when it was compared the lesion sizes of animals receiving different doses of the diaryl compound (D1, D2 and D3) no difference among them was detected $(p>0.05)$. Comparing the groups that received the reference drug (P1, P2 and P3), a significant difference was noticed in their lesion sizes (Fig. 2) $(\mathrm{p}<$ $0.01)$.

It is interesting to notice that no secondary infection was found in the animals treated with the diaryl derivative, what could be explained by the fact that these classes of compounds are good anti-bacteria and anti-fungi drugs (Araújo \& Leon 2001).

The studied compound contain a phenolic group in its structure (Fig. 1) and data from Mukhopadhyay et al. (1982) suggested that this group must be essential for a good biological activity, in accordance with our results of efficiency as well in vitro as in vivo. Also, Venkatesan and Rao (2001) studying a number of ring substituted analogues of curcumin in lipid peroxidation observed that the phenolic derivatives were more active than the nonphenolic analogues, some of which were inactive.

It is very hard to make any kind of association between the in vitro and in vivo results, since we do not know which possible change these compounds undergo inside the vertebrate host. Recent data (Lin et al. 2001) showed that curcumin is first biotransformed to di-

A<smiles>COc1ccc(/C=C/C(=O)/C=C(O)/C=C/c2ccc(O)c(OC)c2)cc1</smiles>

B<smiles>N=C(N)c1ccc(OCCCCOc2ccc(C(N)=NCCCO)cc2)cc1</smiles>

Fig. 1: chemical sctructure of the diaryl derivative (A) and Pentamidine Isethionate (B)

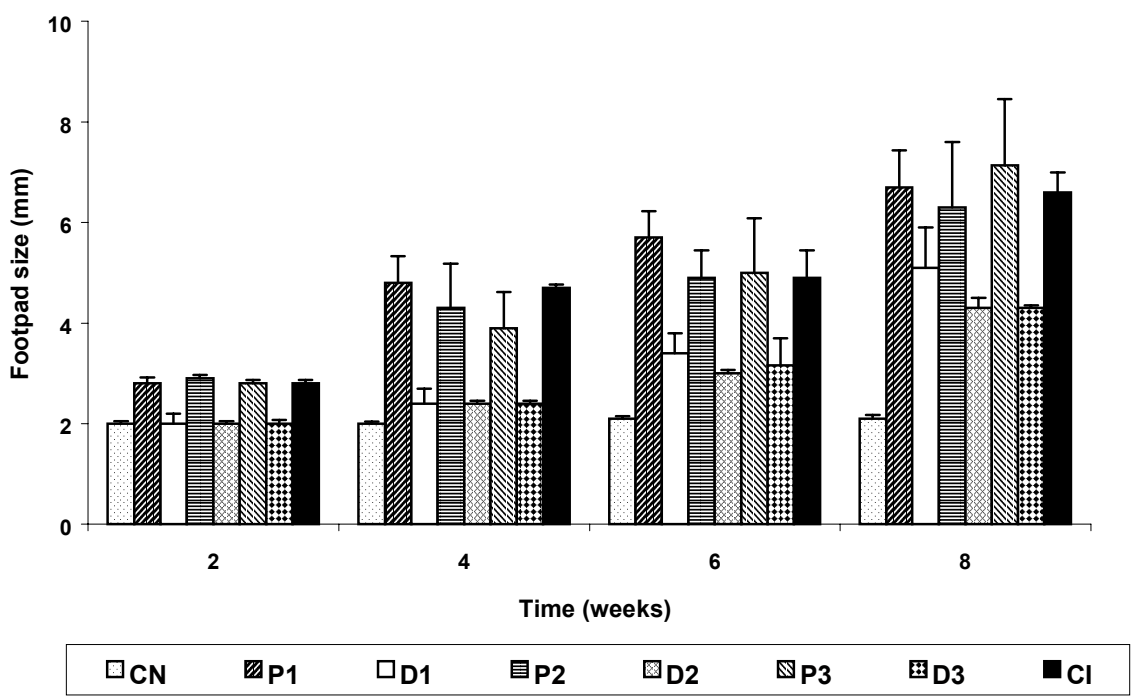

Fig. 2: the in vivo activity of [1-(4-methoxy-phenyl)-7-(3,4-methoxy-4-hydroxy-phenyl)-1, 6-heptadien- 3, 5-dione] 
hydrocurcumin and tetrahydrocurcumin to be active as anti-inflammtory and anti-tumor drug.

\section{REFERENCES}

Alves LV, Canto-Cavalheiro MM, Cysne-Finkelstein L, Leon LL 2003. In vitro antiproliferative effects of several diaryl derivatives on Leishmania sp. Biol Pharm Bull (in press).

Araujo CAC, Leon LL 2001. Biological activities of Curcuma longa L. Mem Inst Oswaldo Cruz 95: 723-728.

Araujo CAC, Alegrio LV, Gomes D, Lima ME, Leon LL 1999. Studies on the effectiveness of curcumin derivatives against Leishmania amazonensis. Mem Inst Oswaldo Cruz 94: 791794.

Araujo CAC, Alegrio LV, Leon LL 1998. Anti-leishmanial activity of compounds extracted and characterized from Centrolobium sclerophyllum. Phytochemistry 3: 751-754.

Desjeux P 2001. The increase in risk factor for leishmaniasis worldwide. Trans $R$ Soc Trop Med Hyg 95: 239-243.

Gomes DCF, Alegrio LV, Lima ME, Leon LL, Araujo CAC
2002. Synthetic derivatives of curcumin and their activity against Leishmania amzonensis. Arznforschung/Drug Research 52: 120-24.

Koide T, Nose M, Ogihara Y, Yabu Y, Ohta N 2002. Leishmanicidal effect of curcumin in vitro. Biol Pharm Bull 25: 131-133.

Lin JK, Pan MH, LinShiau SY 2001. Recent studies on the biofunctions and biotransformations of curcumin. BioFactors 13: 153-158

Mukhopadhyay A, Basu N, Ghatak N, Gujral PK 1982. Antiinflammatory and irritant activities of curcumin analogs in rats. Ag Actions 12: 508-515.

Saleheen D, Ali SA, Ashfaq K, Sissiqui AA, Aga A, Yasinzai MM 2002. Latent activity of curcumin against leishmaniasis in vitro. Biol Pharm Bull 25: 386-389.

Venkatesan P, Rao MN 2001. Structure-activity relationships for the inhibition of lipid peroxidation and the scavenging of free radicals by synthetic symmetrical curcumin analogues. J Pharm Pharmacol 52: 1123-1128. 